\title{
A Review on Sensing of Spectrum in Cognitive Radio Networks
}

\author{
Rahul Prakash ${ }^{1}$ \\ M. Tech Scholar, ECE Dept. BTKIT Dwarahat, Almora Uttrakhand ${ }^{1}$
}

\begin{abstract}
The major requirement for communication is the Bandwidth which is very limited. And for effective utilisation of the bandwidth Cognitive Radios (CR) are used which are is also called Software Defined Radios with inbuilt intelligence, which are used to reduce the burden in the electromagnetic Spectrum. The cognitive radio efficiently allocates the License Bands to Unlicensed user when the licence band is not being used the licensed user which is called the primary user (PU) and the Cognitive Radio user which is called the Secondary User (SU) which may co-exist together in the spectrum bandwidth provided that the secondary user does not interfere with the primary user in the communication process. This is done by the process of Spectrum Sensing which is the very important part for any Cognitive Radio and for the Sensing various techniques are used. This paper presents the concept of Cognitive Radio network and the review of the two techniques which are Cooperative and Non-Cooperative spectrum sensing techniques the paper also highlights the main advantage as well as disadvantage of cognitive radio networks and its future scopes.
\end{abstract}

Keywords: PU (Primary user), SU(Secondary User), CRN(Cognitive Radio Network), Spectrum Prediction, Activity Modelling, CR(Cognitive Radio)

\section{INTRODUCTION}

The idea of cognitive radio firstly conceived by an article published by J. Mitola III and Gerald Q. Maguire ,Jr. in $1999^{[1]}$ cognitive radio will be the next effective radio communication system after software defined Radio this radio spectrum utilise the frequency of $3000 \mathrm{~Hz}$ to $300 \mathrm{GHz}$. By using Advanced Signal Processing technique, it is possible to implement a radio that can actually sense the frequencies which are free to be utilised. As the definition of CR. "A cognitive radio can be defined as a radio device that is aware of its surroundings, and the internal state and with a knowledge of these elements and with any stored pre-defined objectives it can make and implement decisions about its behaviour." ${ }^{[2]}$.

The key feature of the Cognitive Radio is the sensing of spectrum and opportunistically detect the underutilized spectrum which is formally licence to a Primary User (PU) by using the method such as matched filter energy detection and cyclostationary feature detection. The Cognitive Radio networks (CRNs) detects the voids (holes) of the spectrum and uses them for Secondary User (SU) data transmission without any interference with the Primary User (PU) as stated by Z.Quan et al. and A.H. Sayad $2009^{[3]}$ Performance of cognitive radio network is specified regarding the False alarm and the missed detection where False alarm in the specified as where cognitive radio user should have declared the channel is occupied but it is in turn free.

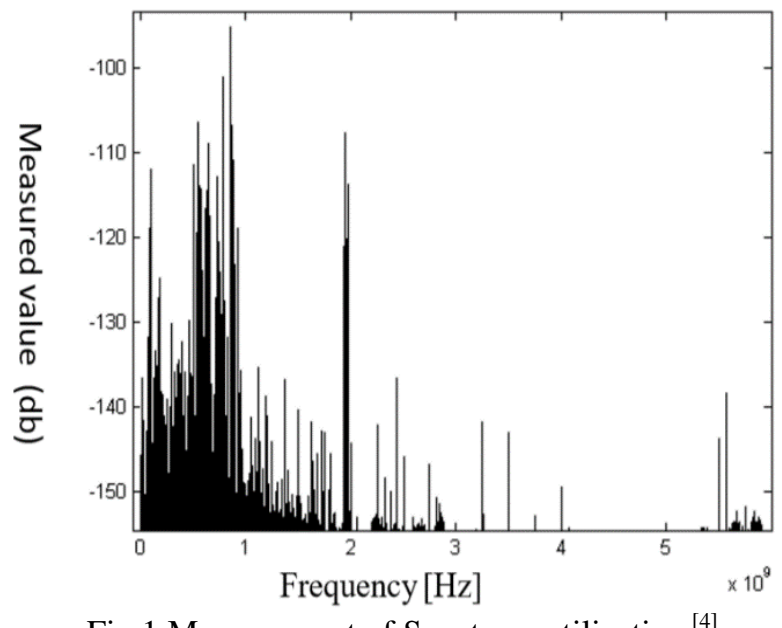

Fig.1 Measurement of Spectrum utilization ${ }^{[4]}$ 


\section{International Advanced Research Journal in Science, Engineering and Technology}

Vol. 5, Issue 11, November 2018

For example (Federal Communication Commission) in the USA is the prime authority which allocates and regulates the frequency spectrum. The frequency chart above shows the spectrum allocated over the complete frequency band which shows a kind of spectrum scarcity especially for the band under the $3 \mathrm{GHz}$ frequency band.

These measurements were taken in a town of Berkeley USA which reveals a distinctive utilization of $0.5 \%$ which further reduces to $0.3 \%$ in the $4 \mathrm{GHz}-5 \mathrm{GHz}$ then which clearly shows that spectrum which is exclusively available for communication is majorly allocated but it is used inefficiently. Therefore, FCC permitted the unlicensed (Secondary User) to use the spectrum in the absence of licensed Primary User as a Secondary User.

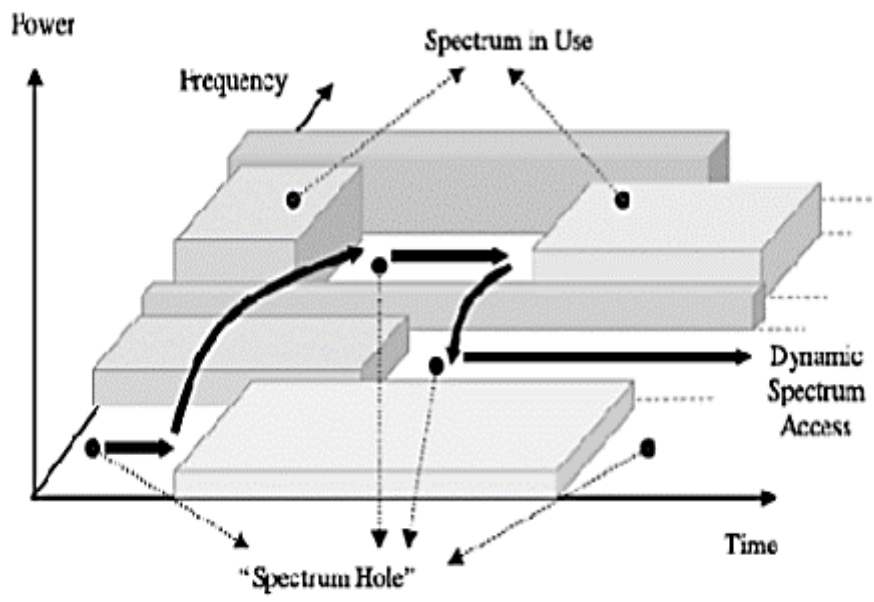

Fig. 2 Spectrum White Spaces (Holes) ${ }^{[5]}$

The under-utilized unused frequency bands are shown as the white spaces ${ }^{[5]}$ in the above figure. Cognitive Radios can change their transmitter parameters based upon the surrounding in which they operate.

\section{TUNCTIONS PERFORMED BY COGNITIVE RADIO NETWORKS}

The two types of user in CRN are Primary User and Secondary User the SU are those users which uses the spectrum while it is not being used by Primary User, if a primary user appear while secondary user is still in the middle of a communication then the secondary user must leave the current spectrum and search for another channel in the spectrum which is empty to continue its communication. The below figure shows various activity performed by a CR user.

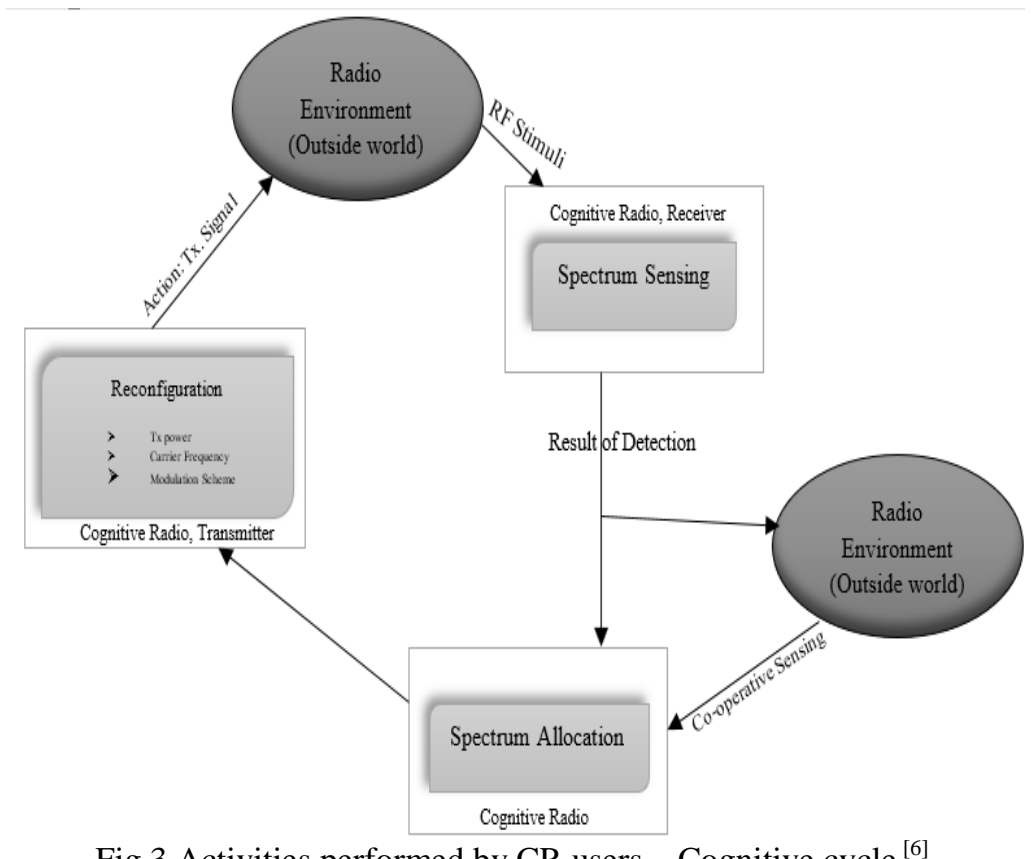

Fig.3 Activities performed by CR users Cognitive cycle ${ }^{[6]}$ 


\section{International Advanced Research Journal in Science, Engineering and Technology}

Vol. 5, Issue 11, November 2018

2.1 Spectrum Sensing: Provides a fair Spectrum Scheduling method which decides which Secondary User can use the spectrum whole at a particular time. The secondary user needs to detect the existence of the primary user in the licensed spectrum and leave the frequency band of the spectrum quickly when any primary user in mergers and to avoid any interference with the primary user it is the first step to implementing CR

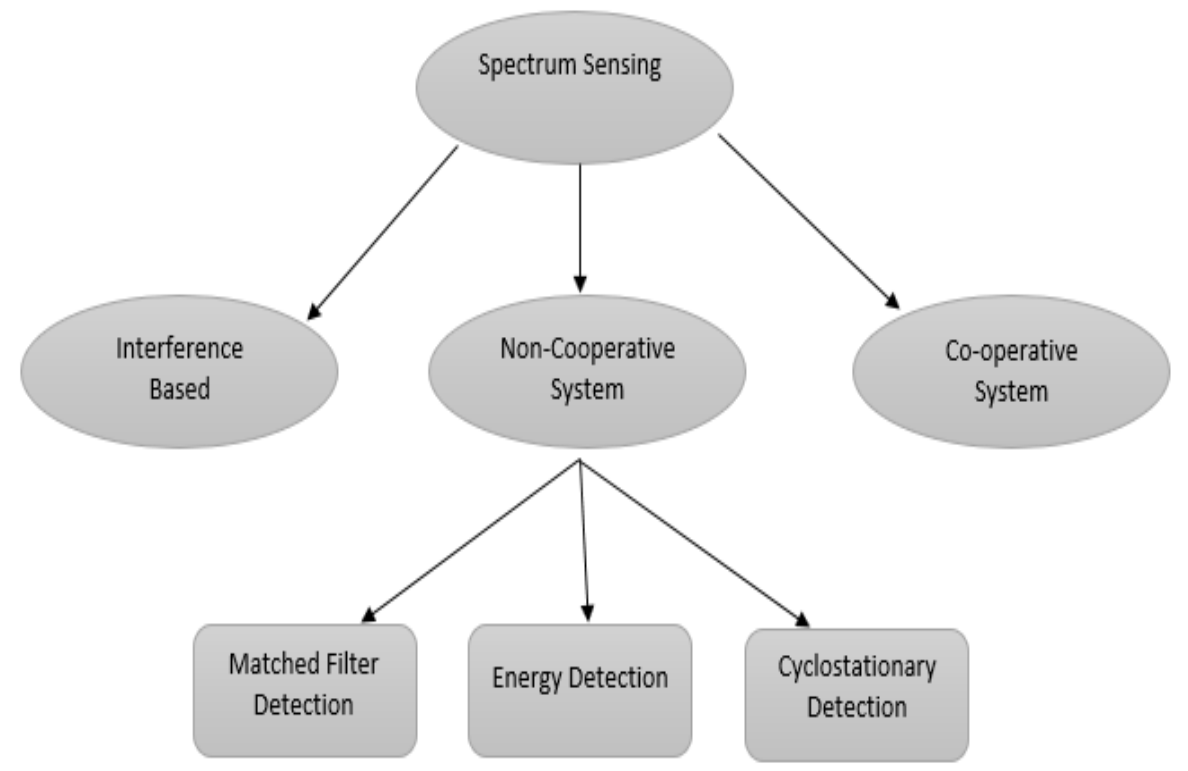

Fig.4 Spectrum Sensing types

2.2 Spectrum Decision: The capability of the Secondary User or a licenced used to detect the best spectrum available according to the quality of service of the available channel it involved spectrum selection spectrum characterization and reconfiguration function. Each vacant channel is characterized by some statistical information and local observation based on the data provided by SU most suitable channel is selected. Characterization of the bands is done on the basis of interference and strength of the received signal and number of users which are currently presents in the channel. This selection is done by the Centralized CRs and Distributed CRs.

2.3 Spectrum Sharing: Spectrum sharing is to distribute the voice of spectrum equally or fairly among the Secondary User. After detecting the available spectrum, the next challenge will be the appropriate channel among the Secondary user according to the cost of uses and requirement. It can be classified by three main aspects. ${ }^{[7]}$

2.3.1 Architecture- tells whether the spectrum access in cognitive radio is distributive or centralized. In the central spectrum sharing the spectrum allocation measurements are forwarded to a centralized entity. Allocation of spectrum is controlled by the centralized entity. In distributed spectrum sharing no Centre authority exists rather each and every node or user allocates and give access to the spectrum.

2.3.2 Behaviour of spectrum allocation-Determines whether it is a cooperative behaviour or non-cooperative behaviour. The Cooperative spectrum sharing takes into account the effect of Communication of one node to another but NonCooperative spectrum sensing consider only the node which is in hand.

2.3.3 Spectrum Accessing Method- Determines whether it is overlay or underlay method. In Overlay method the secondary user access via the channel which are currently not in use of Primary User. In underlay the secondary user access the spectrum by using spread spectrum techniques but in this method the secondary user may get interfered with the Primary User.

2.4 Spectrum Mobility: It is a process when the Cognitive Radio user switches its frequency of operation. When a licensed user starts to get access to the channel that is currently occupied by the secondary user or a licensed user so the second division have to switch to an idle or free frequency band. This process leads to the spectrum hand-off, The functions of spectrum mobility includes -

(i) Spectrum Hand-off

(ii) Connection Management

Spectrum Hand-off can occur if -

(i) Primary user is detected

(ii) Secondary user lost connectivity

(iii) Channel does not meet the QoS requirement 


\section{International Advanced Research Journal in Science, Engineering and Technology}

Vol. 5, Issue 11, November 2018

Types of Hand-offs-

(1) Proactive Hand-off - The Secondary user selects the target channel Before any kind of Transmission so that they could perform handoff if any unwanted condition evolves

(2) Reactive Hand-off - Target channel is selected when the failure in connection occurs, rather than selecting target channel previously.

\section{PHYSICAL ARCHITECTURE OF CR-}

Cognitive radio consists of a transceiver which is equipped with an RF front end and baseband Signal Processing Unit which perform the process of modulation and demodulation and encoding or recording function. ${ }^{[8]}$ Components of $\mathrm{CR}$ are as follows-

3.1 Radio Frequency filter- Used to select only the frequency of interest and discard the undesired spectrum.

3.2 Low Noise Amplifier- Used to amplify the incoming signal and also plays a vital role in determining the noise figure of the system

3.3 Mixer- Is used to do the frequency translation and the RF frequency is converted into the intermediate frequency because processing the signals in lower frequency is much easier and designing of filter is also possible in lower frequencies.

3.4 PLL (Phased Locked Loop) \& VCO- The VCO generates a signal with a fixed frequency by using feedback which is used for mixing.

PLL make sure that the frequency remains constant with time by using a feedback loop.

3.5 Channel Selection Filter- Used to select the appropriate band for communication and reject the adjacent bands

3.6 AGC (Automatic Gain Control)-It is used to keep the level of output constant for a wide range of the input signals.

3.7 A/D Conversion- It samples then Quantize and encode the Analog signal therefore converting it to a digital signal so that it can be processed by the Baseband processing unit.

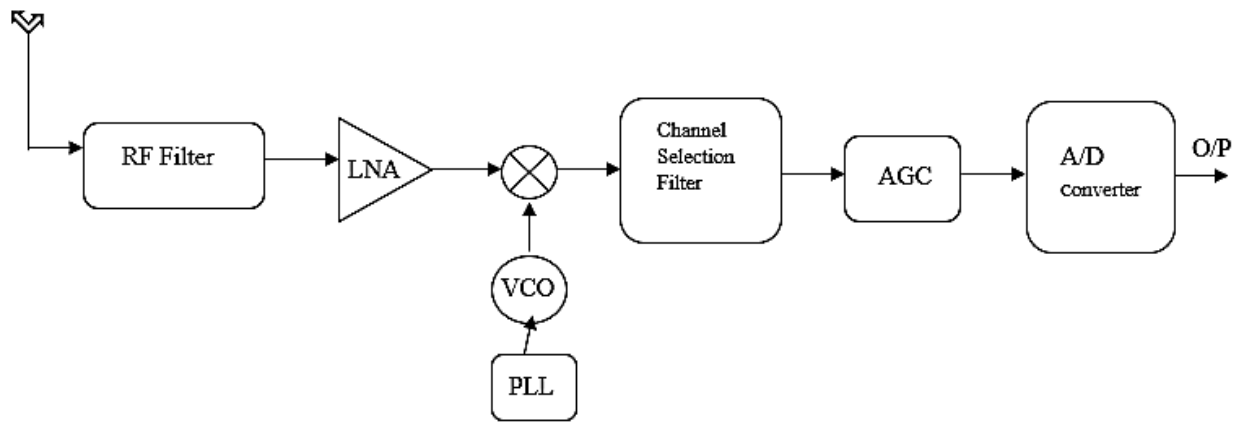

Fig.5 RF front End of a Receiver

\section{SPECTRUM SENSING TECHNIQUES}

The most important tasks of cognitive radio is the Spectrum Sensing and its main feature is the ability to sense the radio which can easily differentiate between PU signal and noise signal it is done to avoid the interference between the Primary User or Secondary User to main categories of spectrum sensing techniques are described below-

4.1 Co-operative Detection (Receiver detection method)- It is a type of centralized detection which can be undertaken by the different radios which shares a common radio network a cognitive radio network. In this is Central node receives the data from all the nodes within the network and then provide a combined decision for a particular channel as this decision is considered as more accurate than individual decision then it is broadcasted.

\section{Advantage}

(i) provide a way to tackle noise, fading etc

(ii) Reduces the probability of False Detection \& False alarm.

(iii) Decrease the Sensing time.

(iv) More accurate for primary user detection

\section{Disadvantages}

(i) More Complex

(ii) Increase the overall traffic overhead

(iii) Always required a control channel 


\section{International Advanced Research Journal in Science, Engineering and Technology}

Vol. 5, Issue 11, November 2018

Types of Cooperative sensing -

(1) Centralized Co-operative sensing

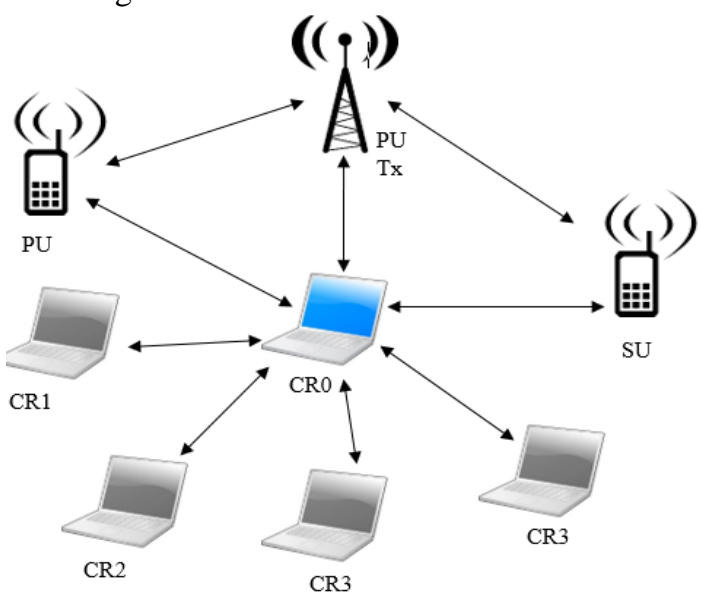

Fig.6 Centralized Cooperative Sensing

In this method one central node is responsible for detection of the primary user signal which is present in the spectrum.

(2) Distributed Co-operative Sensing-

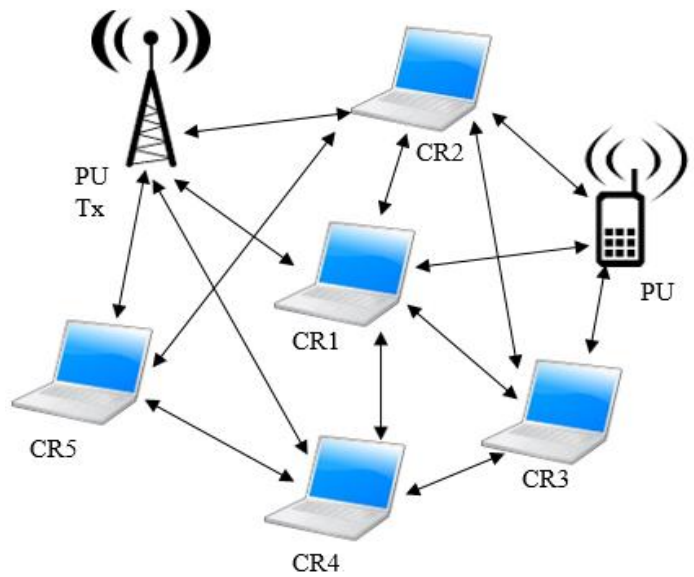

Fig.7 Distributed Cooperative Sensing

This type of network can be build up by not having a need of any centralized node to detect the signal of PU.

\subsection{Non-cooperative Spectrum sensing Technique (Transmitter Detection method)-}

In this the sensing process is done by individual secondary user cognitive nodes these decisions based on Sensed energy from the received signal these are used in order to reduce the interference from primary or licence User. Types of Noncooperative detection techniques-

4.2.1 Energy Detection - It is the simplest non-coherent detection technique, it does not require any prior knowledge about the primary user signal which can also be termed as blind signal detection. ${ }^{[10]}{ }^{[11]}$ It only works with the energy of received signal to decide if it is from a noise source or from a primary user for making that decision with the threshold value is chosen it can be shown by the below block diagram. ${ }^{[12]}$

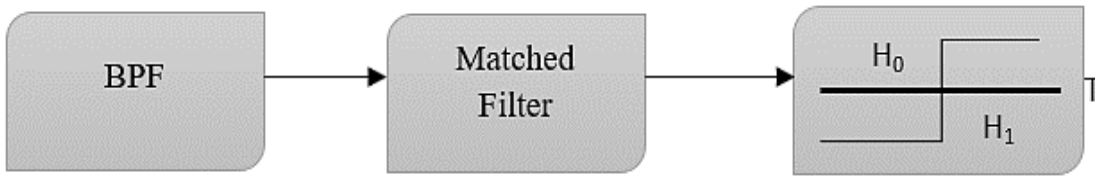

Fig.8 Block Diagram of Energy Detection

4.2.2 Matched Filter Detection (Coherent Detection) - It is the most optimal method for the detection of primary user in the spectrum when we have the knowledge of transmitted signal. Matched filter is used to maximize the SNR in the presence of Additive White Gaussian Noise. ${ }^{[13]}$ 


\section{International Advanced Research Journal in Science, Engineering and Technology}

Vol. 5, Issue 11, November 2018

The Secondary User have the priori knowledge of the Unknown Signal is done with the Filter whose impulse response is time shifted \& then mirrored with respect to desired Signal.

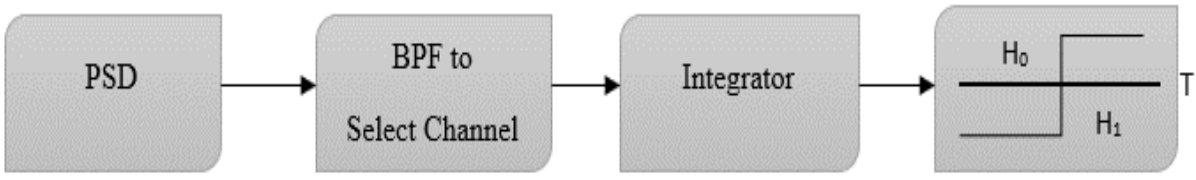

Fig.9 Block Diagram of Matched Filter Detection

Transfer function of Matched Filter can be expressed as-

$\mathrm{x}=$ unknown Signal (Vector)

$$
\sum_{k=-\infty}^{\infty} h[n-k] x[k]
$$

$\mathrm{h}=$ Impulse response of the Matched filter that is matched to reference signal is convolved with it for max SNR

Advantage- (i) Needs less detection time.

(ii) Maximized SNR.

Disadvantages - Prior Knowledge of PU signal required.

\subsubsection{Cyclo-Stationary Features Detection-}

It is a method in which the periodicity property is exploited of the received signal from the Primary User. Periodicity is present in sinusoidal Carriers pulse terrains and spreading codes these all are called Cyclostationary signals which exhibits spectral correlation which is generally not found in noise and interference signal. ${ }^{[13]}$

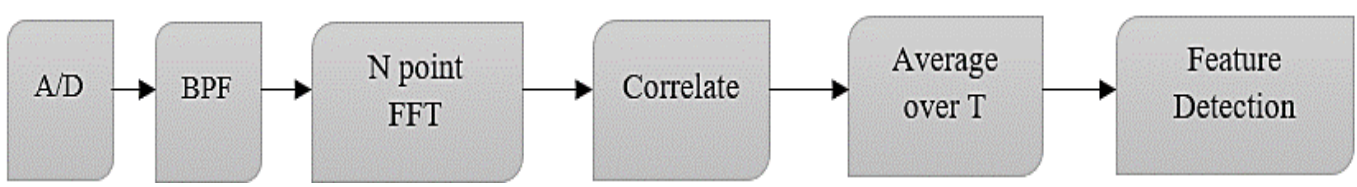

Fig.10 Block Diagram Cyclo-stationary feature detection

Advantages- (i) Perform in a better way than energy detection in the low SNR region

(ii) Eliminates the Synchronization requirement

(iii) Improve the overall CR throughput

Disadvantages-

(i) High Computational Complexity.

(ii) Long Sensing time.

(iii) Only detect selective primary signal with some Characteristics.

Graph shown below determine the trade-off for sensing and complexity of various spectrum sensing method

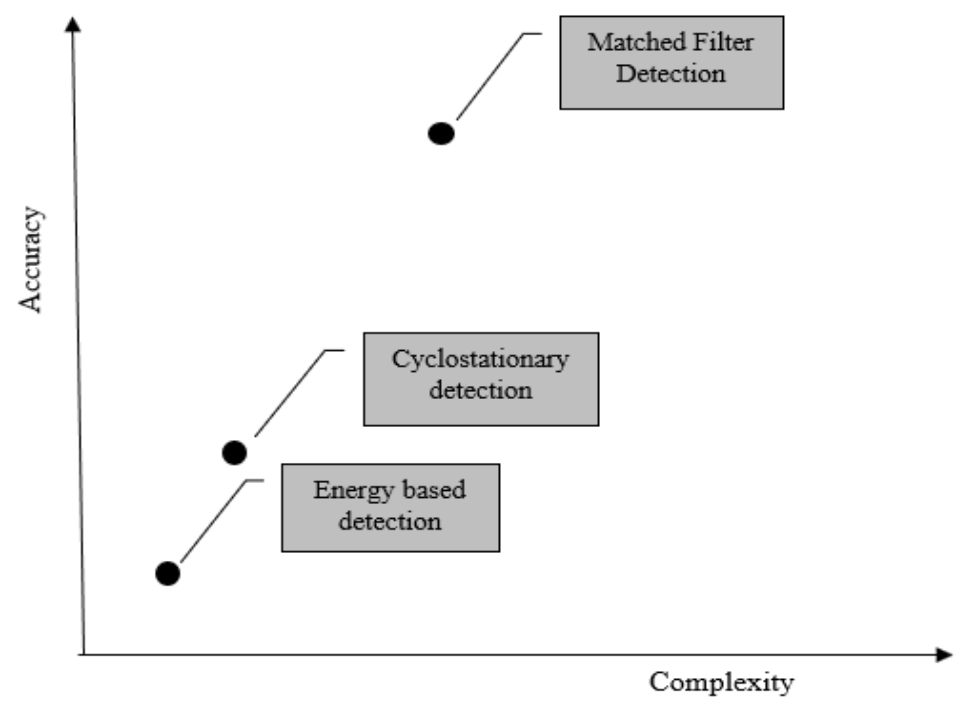

Fig.11 sensing complexity Vs accuracy graph 


\section{International Advanced Research Journal in Science, Engineering and Technology}

Vol. 5, Issue 11, November 2018

\section{CHALLENGES IN SPECTRUM SENSING}

(i) Detection of the licensed primary user in a very short time duration as it is required for developing the interference model by measuring the interference temperature. ${ }^{[14]}$

(ii) The required SNR which is to be detected may be very low.

(iii) As multipath fading and the time Dispersion of the channel complicates the sensing process.

(iv) The noise or interference present in a signal may change with time and location Which result noise power uncertainty for detection process.

(v) For selecting the sensing techniques trade-off should be considered such as the accuracy, complexity, sensing duration, requirement of channel and network requirement.

\section{CONCLUSION}

As we know that the spectrum of frequency for communication is a very valuable resource in wireless communication. And the cognitive radio network is the most promising and efficient technology to utilise the frequency spectrum completely for past application which are useful for wireless communication. In this paper, we have tried to give a brief and concise introduction to the architecture and function of the cognitive radio like the spectrum sensing which is the most important and major task of Cognitive Radio Networks. We have also discussed some spectrum sensing technique with their respective advantages and disadvantages and we also saw that the matched filter detection is the most efficient way to send the spectrum till now for the primary user signal detection.

\section{REFERENCES}

[1]. J. Mitola, III,G. Q. Maguire, Jr., "Cognitive radio: making software radios more personal", Personal Communications, IEEE [see also IEEE Wireless Communications], vol. 6, pp. 13, 1999.

[2]. [online] Available: http://www.radio-electronics.com/info/rf-technologydesign/cognitive-radio-cr/technologytutorial.php

[3]. Z. Quan, S. Cui, A. Sayed, and H. V. Poor, "Optimal multiband joint detection for spectrum sensing in cognitive radio networks," IEEE Transactions on Signal Processing, vol. 57, no. 3, pp. 1128-1140, March 2009.

[4]. R. Thomas, D. Friend, L. Das and 2006, "Cognitive networks and learning to achieve end-to-end performance objectives", IEEE Communications Magazine, vol. 44, no 12, pp. 51-57

[5]. Simon Haykin, D.J Thomson, and Jeffrey H. Reed (2009), "Spectrum Sensing for Cognitive Radio", IEEE Proceeding, Vol. 97, No.5, pp: 849-877.

[6]. Singh, Ashutosh, and Varsha Saxena. "Different spectrum sensing techniques used in non-cooperative system." International Journal of Engineering and Innovative Technology (IJEIT) Volume 1

[7]. V. U. Kanth, K. R. Chandra, and R. R. Kumar, April 2013, "Spectrum Sharing In Cognitive Radio Networks", (IJETT) - Vol.4 Issue 4.

[8]. I.F. Akyildiz, Won-Yeol Lee, Mehmet C. Vuran, and S.Mohanty, "NeXt generation/dynamic spectrum access/cognitive Radio Wireless Networks: A Survey,"

[9]. MOHD.Sajid Imam, Sheetal Ingle, ShabnamAra, "A Review paper based on spectrum sensing techniques in Cognitive Radio Networks", IISTE, Network and Complex Systems, ISSN 2224-610X (Paper) ISSN 2225-0603 (Online), Vol.3, No.9, 2013.

[10]. I.E.Igbinosa, Olutayo O. Oyerinde, Viranjay M. Srivastava, Stanley Mneney, "Spectrum Sensing Methodologies for Cognitive Radio Systems A Review", International Journal of Advanced Computer Science and Applications (IJACSA), Vol. 6, No. $12,2015$.

[11]. Ian F. Akyildiz, Won-Yeol Lee, Mehmet C. Vuran, Shantidev Mohanty, "Next generation/dynamic spectrum access/cognitive radio wireless networks: A survey", ELSEVIER, I.F. Akyildiz et al. / Computer Networks 50 (2006) 2127-2159.

[12]. B.Mounika, Kolli Ravi Chandra, Rayala Ravi Kumar, "Spectrum Sensing Techniques and Issues in Cognitive Radio", International Journal of Engineering Trends and Technology (IJETT) - Volume4 Issue4- April 2013.

[13]. A. Sahai, N. Hoven, and R. Tandra, "Some fundamental limits in cognitive radio," in Proceedings of the Conference on Communication, Control, and Computing, Monticello, Ill, USA, 2004

[14]. N.Yadav, S.Rathi "Spectrum Sensing Techniques: Research, Challenge and Limitations" IJECT Vol. 2, IssuE 4, OCT. - DEC. 2011 